\title{
INCIDENCIA DEL POLEN DE QUERCUS EN LA ATMÓSFERA DE MÁLAGA Y SU RELACIÓN CON LOS PARÁMETROS METEOROLÓGICOS
}

\author{
Marta RECIO, M. Mar TRIGO, F. Javier TORO y Baltasar CABEZUDO
}

\begin{abstract}
RESUMEN. Incidencia del polen de Quercus en la atmósfera de Málaga y su relación con los parámetros meteorológicos. En este trabajo se presentan los resultados aerobiológicos obtenidos en Málaga para el tipo polínico Quercus, tras el muestreo realizado durante los años 1992-1998 por medio de un captador volumétrico de tipo Hirst. Se analizan los comportamientos estacionales e intradiarios que ha presentado cada año, así como su relación con los principales parámetros meteorológicos. Asimismo, se comparan estos resultados con los obtenidos en otras ciudades españolas y europeas. El polen de Quercus se detecta en la atmósfera de Málaga fundamentalmente durante los meses de Marzo, Abril y Mayo, registrándose las máximas concentraciones generalmente en Abril, con la excepción de 1997, en que se adelantó a Marzo. Se trata de un taxon que durante un buen número de días alcanza concentraciones de altas (50-100 granos/ $\left.\mathrm{m}^{3}\right)$ a muy altas $\left(>100\right.$ granos $\left./ \mathrm{m}^{3}\right)$, habiéndose llegado a obtener concentraciones medias diarias de hasta 1578 granos/ $\mathrm{m}^{3}$ durante 1997. Las cantidades totales de polen detectadas fueron muy variables de un año a otro, sin embargo, respecto al resto de los tipos polínicos que se registran en Málaga, el polen de Quercus casi siempre ocupa el tercer puesto en orden de abundancia anual, después del de olivo y del de cupresáceas. En cuanto a distribución intradiaria no presentan un comportamiento estable aunque, en general, ha mostrado una ligera tendencia a aumentar durante la noche y la madrugada. Las correlaciones obtenidas entre los valores diarios de concentración atmosférica de polen y diferentes parámetros meteorológicos demuestran que, en Málaga, la presencia de este tipo polínico siempre se asocia positivamente con parámetros indicadores de calor (insolación y temperatura), velocidad y dirección noroeste del viento y, negativamente, con la frecuencia de calma y del resto de las direcciones de vientos. Estas correlaciones obtenidas con viento parecen indicar que la mayoría de los pólenes de Quercus que se registran en Málaga proceden de las sierras del interior, donde se encuentran las mejores poblaciones de encinas, coscojas y alcornoques, siendo arrastrados hacia la capital costera por los vientos del noroeste. Al igual que ocurriera en otras estaciones de la Red Española de Aerobiología, 1997 fue el año con polinación máxima y más adelantada. A ello parece ser que contribuyeron las intensas lluvias otoñales previas al período polinación, con la consiguiente formación de numerosas yemas florales, así como las altas temperaturas que se alcanzaron durante el invierno (Febrero-Marzo), que favorecieron la maduración y apertura de la mayoría de las yemas florales formadas.
\end{abstract}

Palabras clave. Aerobiología, alergia, polen, Quercus, Málaga, fenología, meteorología.

ABSTRACT: Airborne pollen of Quercus in Malaga (S. Spain) and its relationship with meteorological parameters. In this work we present the airborne pollen measurements of Quercus in Málaga during the 1992-1998 period. The study was carried out whit the aid of a volumetric spore-trap (Burkard seven-day recorder) located on the flat roof of the Faculty of Medicine, $1 \mathrm{~km}$ far from the city centre. We have also

Este trabajo ha sido financiado con cargo a los proyectos PB92-0814-02 de la DGICYT y AMB970457-C07-05 del Plan Nacional I+D de la CICYT. 
analysed the seasonal and intradiurnal variations and their relationship with the meteorological parameters by means of the Spearman's correlation test. This pollen type was mainly detected in the atmosphere of Málaga during March, April and May, with a maximum peak in April, excluding 1997, in which the peak take place in March. The mean daily pollen concentrations are high (50-100 pollen grains/cubic meter of air) to very high ( $>100$ pollen grains/cubic meter of air) during several days, the maximum daily value detected reaching 1578 pollen grains/ $\mathrm{m}^{3}$ in 1997 . The annual total concentrations were different for the different years studied, however the Quercus pollen always became the third with relation to the others pollen types detected in Malaga. The intradiurnal variation patterns were not stable from year to year, however, in general, this taxon lightly increased at night. The correlations obtained between daily pollen concentrations and different meteorological parameters showed that the presence of this pollen type in the air of Malaga is positively associated with sunshine, temperature, speed and northwesterly wind direction; and negatively associated with calm frecuency and the others wind directions. These positive and significant correlations obtained with northwesterly wind show that the Quercus pollen registered in Malaga comes from inlands where the holm oaks, cork trees, and kermes oaks are common. In Malaga, as that occured in others stations of the Spanish Aerobiological Network (the REA), 1997 was the year when the earliest maximum peak and the higest concentrations were obtained. We think the abudant rainfalls occurred during the previous autumn contributed to the formation of high quantities of flowering buds in Quercus, and the warm temperatures detected during the following winter (February and March) to the early maduration and opening of they.

Key words. Aerobiology, allergy, pollen, Quercus, Malaga, phenology, meteorology.

\section{INTRODUCCIÓN}

El género Quercus está representado en la provincia de Málaga por encinas $(Q$. rotundifolia Lam.), alcornoques (Q. suber L.), coscojas ( $Q$. coccifera L.), quejigos ( $Q$. faginea Lam., Q. canariensis Wild. y $Q$. alpestris Boiss.) y melojos (Q. pyrenaica Wild.), siendo las especies más abundantes las encinas, alcornoques y coscojas. Las primeras son más frecuentes en la zona oriental caliza y seca; los alcornoques viven preferentemente en la zona occidental de la provincia, de suelos ácidos y más lluviosa; mientras que las coscojas tienen una distribución más uniforme predominando en zonas básicas y secas. El resto de las especies son poco abundantes y se distribuyen por lo general en las zonas más húmedas y occidentales o en las zonas más altas de la provincia. Se trata de especies que presentan una alta producción floral y polínica (Hidalgo et al., 19996a, 1996b). Además, debido a esta elevada producción por unidad de superficie y año, el polen tiene gran importancia como alimento para las abejas. No obstante, muchos de sus pólenes son dispersados por el aire al presentar polinización anemófila.

El polen de Quercus es trizonocolporado, isopolar, radiosimétrico, de circular a subtriangular-angulaperturado en visión polar y corte óptico ecuatorial, y de circular a elíptico en visión ecuatorial y corte óptico meridiano. Su tamaño es variable, de pequeño a mediano (10-50 $\mu \mathrm{m}$ de diámetro), siendo generalmente más pequeño en $Q$. coccifera y $Q$. rotundifolia que en las otras especies citadas (Díez \& Suárez, 1987).

El polen de diversas especies de Quercus ha sido citado como alergógeno por distintos autores como Sáenz (1978), Lewis et al. (1983), Halse (1984) y Domínguez Vilches et al. (1984). En general, las personas que sufren problemas de polinosis a este taxon suelen presentar un período largo de disconfort debido a que existereactividad cruzada con el polen de otras especies del mismo género, con el de especies de géneros emparentados como Betula y Castanea, y con el de gramíneas (Eriksson, 1978; Fernández Caldas et al., 1989; Sutra et al., 1990; Ickovic \& Thibaudon, 1991; Ross et 
al., 1996). Por otra parte, Kormuták \& Ostrolucká (1992) encontraron un elevado grado de polimorfismo entre las proteínas de distintas especies de Quercus.

Algunos autores creen que las bajas correlaciones obtenidas entre las cantidades de polen dispersadas por el aire y el número de casos de polinosis se debe a que estas especies del género Quercus presentan un grado de alergenicidad moderado (Lewis et al., 1983). Sin embargo, Nilsson \& Spieksma (1992) consideran que la alergenicidad e incidencia de estos pólenes no ha sido estudiada suficientemente. Por todo ello, y ante el hecho de que en la atmósfera de Málaga se detectan altas concentraciones de polen de este taxon (Cabezudo et al., 1994; Recio et al., 1995, 1998a, 1998b; Toro et al., 1996; Trigo et al., 1998), creemos conveniente realizar un estudio más profundo de este tipo polínico desde el punto de vista aerobiológico, analizando su comportamiento estacional e intradiario y estudiando la posible relación que podrían tener con los diferentes parámetros meteorológicos.

\section{MATERIAL Y MÉTODOS}

El muestreo aerobiológico se ha llevado a cabo mediante un captador volumétrico (Hirst, 1952) modelo seven-day-recording (Burkard Manufacturing Co. Ltd., Rickmansworth, Hertfordshire, England), situado a unos $15 \mathrm{~m}$ del nivel del suelo, en la azotea de la Facultad de Medicina de la Universidad de Málaga. Este edificio se encuentra ubicado aproximadamente a $1 \mathrm{~km}$ al oeste del núcleo urbano y en una zona abierta que permite la libre circulación del aire.

El recuento de los granos de polen se ha realizado según el método recomendado por la Red Española de Aerobiología (Domínguez Vilches et al., 1991; Domínguez Vilches, 1995), presentándose los resultados finalmente como valores medios diarios, expresados en número de granos de polen por metro cúbico de aire $\left(\mathrm{N}^{\circ}\right.$ g.p. $\left./ \mathrm{m}^{3}\right)$.

Se ha determinado el período de polinación principal (PPP) en base al 95\% del polen total anual, utilizando valores acumulados desde el día 1 de Enero (Pathirane, 1975), habiéndose eliminando el 2,5\% inicial y final.

Para analizar la distribución intradiaria del polen atmosférico, se han tomando los recuentos horarios de los días exentos de precipitaciones cuya concentración media diaria era igual o superior a la media del PPP, representándose gráficamente las curvas con los valores porcentuales obtenidos cada dos horas, y calculándose el índice de distribución intradiario (IDI) según la metodología propuesta por Trigo et al. (1997).

El estudio de la relación existente entre los niveles atmosféricos de polen de Quercus y los diversos parámetros meteorológicos se ha realizado por medio del test de correlación no paramétrico de Spearman, utilizándose valores diarios. No se ha utilizado análisis de correlación paramétrica debido a la no normalidad de los datos, aún aplicando diversas funciones logarítmicas. Las variables meteorológicas utilizadas son: horas de sol, precipitaciones (en $\mathrm{mm}$ ), humedad relativa (en $\%$ ), temperaturas media, máxima y mínima (en ${ }^{\circ} \mathrm{C}$ ), velocidad media del viento (en $\mathrm{km} / \mathrm{h}$ ), dirección del viento y calmas (\% de cada uno de los cuatro cuadrantes y de calmas). Estas correlaciones se han realizado tomando todos los días del PPP, sin dividirse en periodos prey post-pico, como habitualmente venimos realizando (Recio et al., 1996, 1997; Toro et al., 1998a), ya que la curva polínica de este taxon presenta varios picos importantes a 10 largo de la estación.

Los datos meteorológicos han sido cedidos por el Centro Meteorológico Territorial de Andalucía Oriental, y corresponden a la Estación del Aeropuerto de Málaga, situada a unos $5 \mathrm{~km}$ al sur de la estación de muestreo. 


\section{RESULTADOS}

\section{Variación estacional}

El polen de Quercus se detecta en la atmósfera de Málaga fundamentalmente durante los meses de Marzo, Abril y Mayo, período en el que se registra entre el $86 \%$ y el 99\% del total anual (fig. 1 y tab. 1). La duración del período de polinación principal (PPP) ha sido relativamente similar en todos los años estudiados, con una media de 83 días y una desviación stardard de \pm 16 días (tab. 2). Sin embargo, la cantidad de polen total fue muy variable de un año a otro (3714 \pm 2169$)$, oscilando entre los 1647 g.p. $/ \mathrm{m}^{3}$ de 1996 y los 8014 g.p. $/ \mathrm{m}^{3}$ de 1997 . Con respecto al polen total anual, el de Quercus representó entre el $3,9 \%$ en 1996 y el $16,4 \%$ en 1995 (tab. 2).

En general, aunque se producen varios picos a lo largo del PPP, los valores máximos se registran en Abril. Sin embargo, en 1997 tanto el máximo mensual como el diario se adelantaron al mes de Marzo (tabs. 1 y 2). Por otra parte, en 1992, aunque el máximo diario se detectara en Mayo (tab. 2), hay que tener en cuenta que durante Abril se registraron dos picos de similar intensidad (fig. 1), que además son muy bajos en comparación con los que se alcanzan normalmente $\left(>200 \mathrm{~g} \cdot \mathrm{p} . / \mathrm{m}^{3}\right)$. El valor máximo medio para el periodo de estudio fue de 481 g.p. $/ \mathrm{m}^{3}$, y el máximo histórico correspondió a 1997, en que fue de 1578 g.p./ $\mathrm{m}^{3}$. Se trata de un taxon que durante varios días alcanza concentraciones de altas (50-100 g.p./ $\left.\mathrm{m}^{3}\right)$ a muy altas $\left(>100\right.$ g.p. $\left./ \mathrm{m}^{3}\right)$.

\section{Variación intradiaria}

En lo referente a la variación intradiaria, el polen de Quercus no presenta un comportamiento estable, ya que para cada uno de los años estudiados los patrones de distribución intradiaria han sido diferentes (fig. 2). En general, aparece en la atmósfera de
Málaga durante las 24 horas del día, mostrando una ligera tendencia a aumentar durante la noche y la madrugada, aunque en 1993 y 1992 se obtuvieron picos hacia el mediodía y por la tarde, respectivamente.

El índice de distribución intradiaria (IDI) de este taxon es muy bajo, siendo su valor medio de $0,08 \pm 0,02$. El valor más alto correspondió al año $1994(0,11)$ y el más bajo a 1992 (0,05). Este comportamiento suele ser indicativo de transporte a larga distancia (Trigo et al., 1997).

\section{Correlación con parámetros meteorológicos}

En la tabla 3 se observa que, en general, los resultados han sido similares para todos los años estudiados. En lo que se refiere a temperaturas e insolaciones, es decir, parámetros indicadores de calor, se han obtenido coeficientes de correlación significativos y positivos, siendo los más significativos los obtenidos con la temperatura máxima. Tan sólo en 1998 se careció de correlaciones significativas.

En cuanto a los vientos, casi siempre se han obtenido correlaciones significativas y positivas con la velocidad y negativas con la frecuencia de calmas. Y con respecto a la dirección, los numerosos coeficientes significativos que se obtuvieron, fueron siempre positivos con los del cuarto cuadrante (centrado en la dirección NO) y negativos con los del resto. En los años 1992 y 1996 no se han obtenido correlaciones significativas con ninguno de los parámetros relacionados con el viento.

La humedad relativa casi siempre ha mostrado correlación significativa y negativa con la concentración de polen atmosférico de Quercus.

Por último, respecto a las precipitaciones, se han obtenido coeficientes de correlación significativamente positivos sólo para los años 1992 y 1995. 

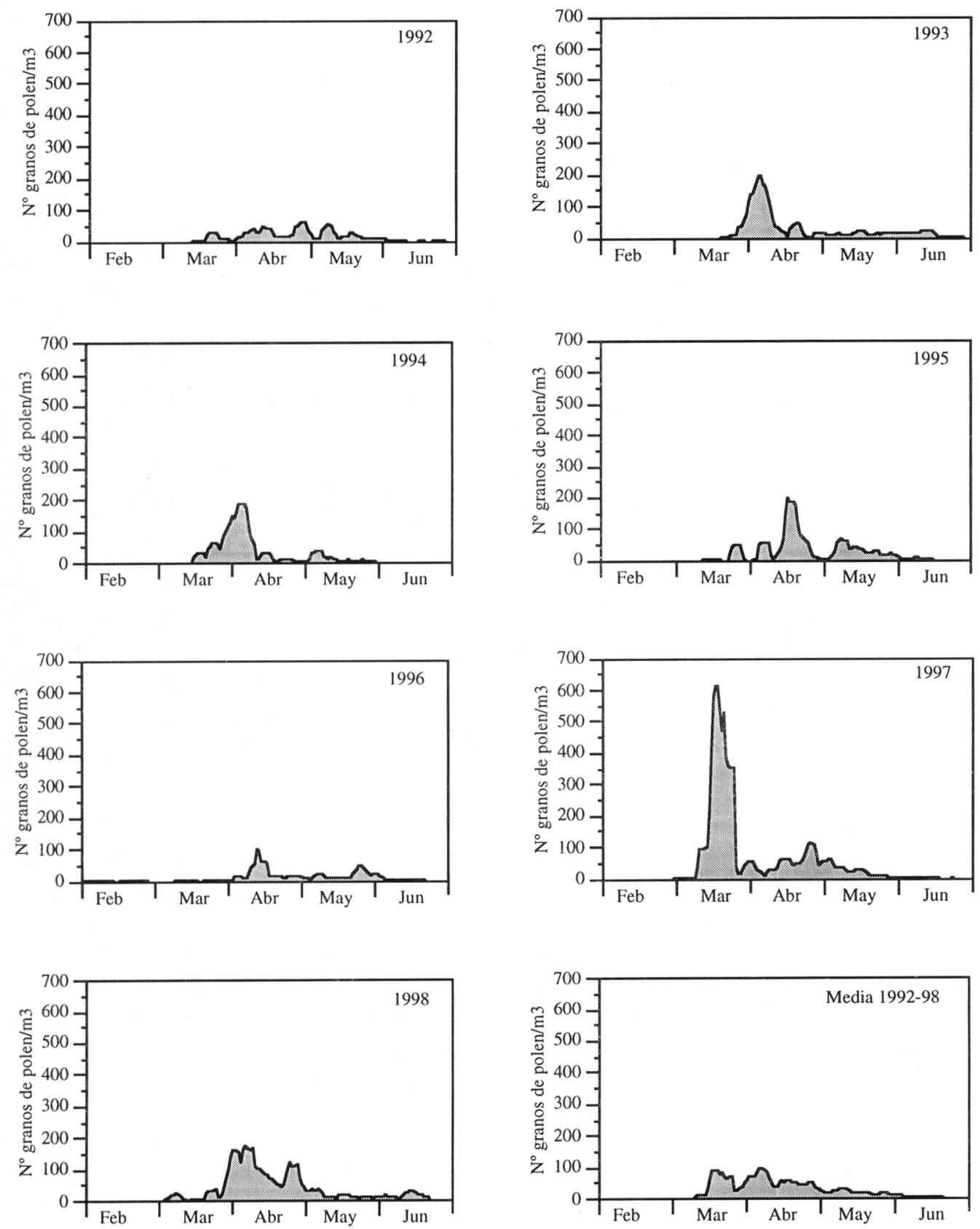

Figura 1. Medias móviles de cinco días de concentración atmosférica de polen de Quercus en Málaga. Five days running means for the concentration of airborne Quercus pollen in Malaga. 


\begin{tabular}{rcccccccccccccc}
\hline & E & F & M & A & M & J & J & A & S & O & N & D & Total & $\%$ \\
\hline 1992 & 0 & 0 & 200 & 904 & 736 & 65 & 11 & 2 & 1 & 2 & 1 & 3 & 1925 & 8,5 \\
1993 & 2 & 0 & 47 & 2146 & 461 & 377 & 18 & 4 & 8 & 5 & 1 & 2 & 3071 & 9,7 \\
1994 & 4 & 4 & 601 & 2092 & 449 & 35 & 9 & 0 & 1 & 0 & 6 & 3 & 3204 & 11,3 \\
1995 & 11 & 6 & 282 & 1856 & 926 & 141 & 23 & 2 & 0 & 0 & 0 & 0 & 3246 & 16,4 \\
1996 & 3 & 59 & 51 & 807 & 554 & 162 & 5 & 0 & 2 & 0 & 3 & 1 & 1647 & 3,9 \\
1997 & 0 & 4 & 5425 & 1669 & 837 & 57 & 5 & 2 & 0 & 5 & 9 & 2 & 8014 & 12,3 \\
1998 & 5 & 5 & 502 & 3350 & 601 & 398 & 20 & 5 & 2 & 0 & 2 & 0 & 4891 & 14,5 \\
\hline
\end{tabular}

Tabla 1. Valores mensuales y anuales de polen atmosférico de Quercus (suma de concentraciones medias diarias expresadas en granos $/ \mathrm{m}^{3}$ de aire) y porcentajes anuales con respecto al polen total, registrados en Málaga durante los años 1992-98. Monthly and annual values of Quercus airborne pollen (sum of the mean daily concentrations expressed in grains $/ \mathrm{m}^{3}$ of air) recorded in Málaga during 1992-98. The last column expresses the annual percentage of Quercus with regard to the total pollen counts.

\begin{tabular}{|c|c|c|c|c|c|c|c|}
\hline & 1992 & 1993 & 1994 & 1995 & 1996 & 1997 & 1998 \\
\hline Fecha del PPP & $\begin{array}{c}23 \mathrm{Mar} \\
\mathrm{a} \\
5 \mathrm{Jun}\end{array}$ & $\begin{array}{c}29 \mathrm{Mar} \\
\mathrm{a} \\
18 \mathrm{Jun}\end{array}$ & $\begin{array}{c}20 \mathrm{Mar} \\
\mathrm{a} \\
27 \mathrm{Abr}\end{array}$ & $\begin{array}{c}28 \mathrm{Mar} \\
\mathrm{a} \\
10 \mathrm{Jun}\end{array}$ & $\begin{array}{c}20 \mathrm{Feb} \\
\mathrm{a} \\
11 \mathrm{Jun}\end{array}$ & $\begin{array}{l}14 \text { Mar } \\
\text { a } \\
20 \text { May }\end{array}$ & $\begin{array}{l}11 \mathrm{Abr} \\
\mathrm{a} \\
16 \mathrm{Jun}\end{array}$ \\
\hline Longitud del PPP ( ${ }^{\circ}$ días) & 75 & 82 & 69 & 75 & 112 & 68 & 98 \\
\hline Máximo diario (granos $\left./ \mathrm{m}^{3}\right)$ & 125 & 346 & 261 & 411 & 225 & 1578 & 419 \\
\hline Fecha del máximo diario & 10 May & $9 \mathrm{Abr}$ & $7 \mathrm{Abr}$ & $20 \mathrm{Abr}$ & $17 \mathrm{Abr}$ & $25 \mathrm{Mar}$ & $8 \mathrm{Abr}$ \\
\hline $\mathrm{N}^{\circ}$ días con $<10$ granos $/ \mathrm{m}^{3}$ & 77 & 96 & 82 & 93 & 106 & 74 & 79 \\
\hline $\mathrm{N}^{\circ}$ días con $11-50$ granos $/ \mathrm{m}^{3}$ & 33 & 46 & 24 & 27 & 30 & 42 & 41 \\
\hline$N^{o}$ días con $51-100$ granos $/ \mathrm{m}^{3}$ & 8 & 2 & 8 & 11 & 3 & 9 & 14 \\
\hline $\mathrm{N}^{\circ}$ días con $>100$ granos $/ \mathrm{m}^{3}$ & 3 & 9 & 11 & 9 & 2 & 15 & 14 \\
\hline
\end{tabular}

Tabla 2. Datos característicos para el polen de Quercus en Málaga durante el período de polinación principal (PPP). Characteristic data for Quercus airborne pollen in Málaga during the main pollen season(PPP): Dates of starting and ending of PPP; length of PPP (No. days); maximum daily pollen count (grains $\left./ \mathrm{m}^{3}\right)$; date of maximum daily pollen count; No. of days with pollen $<10,11-50,51-100$ and $>100$ grains $/ \mathrm{m}^{3} . S D=$ standard desviation.

\section{DISCUSIÓN}

\section{Variación estacional}

En lo que se refiere a duración del PPP, momento y valor de máximas concentraciones, se ha observado que el comportamiento estacional del polen de Quercus en Málaga difiere del de ciudades situadas más al norte de la Península, donde los máximos aparecen más retrasados (Mayo-Junio) y las cantidades detectadas son más bajas (Cabezudo, ed., 1998a, 1998b).
Quercus es un género abundante en la mayor parte de Europa y, aunque son diversas las especies repartidas por este continente, éstas no difieren mucho en la época de polinación. que presentan, obteniéndose un patrón similar de comportamiento en los calendarios polínicos de las diferentes regiones europeas. No obstante, el período de polinación siempre es más tardío en el norte (Mayo-Junio) y centro (Mayo) de Europa que en el sur, donde las máximas concentraciones polínicas se producen en Abril (Spieksma, 1991; Nilsson \& Spieksma, 1992). 


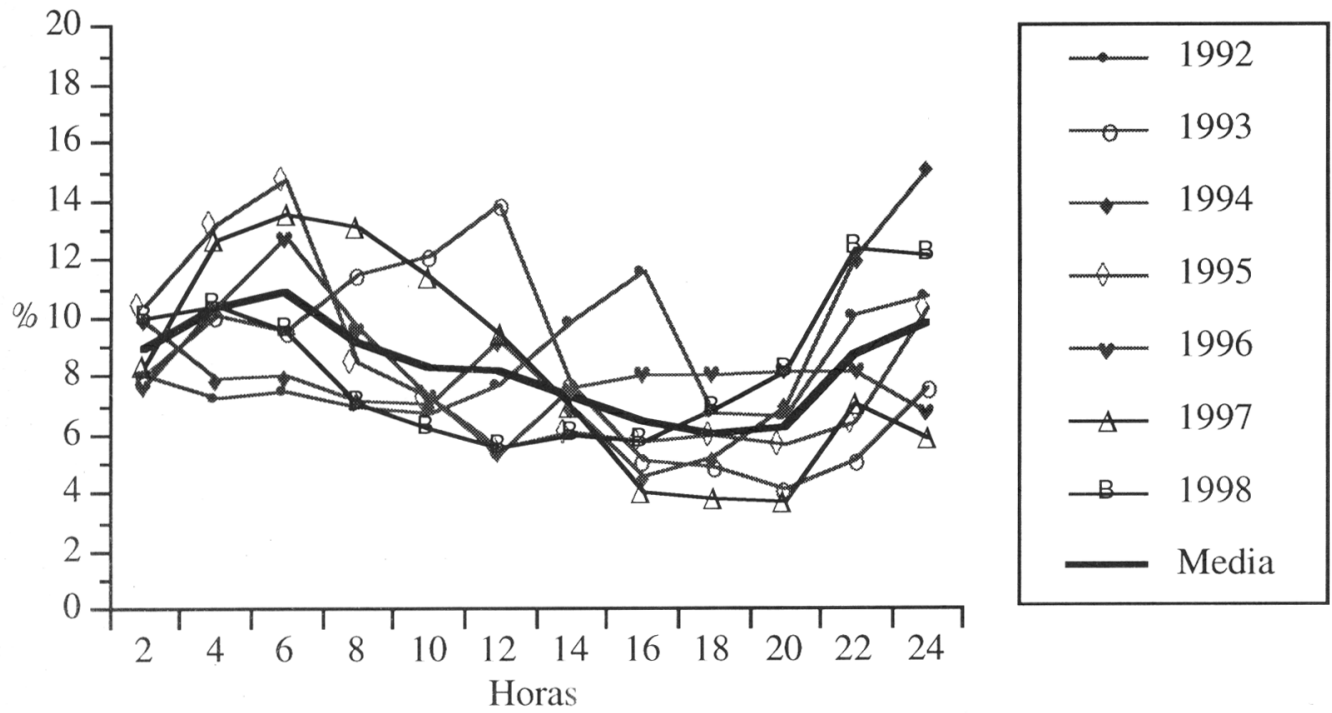

Figura 2. Variación intradiurna de poien atmosférico de Quercus en Málaga. Intradiurnal variation of airborne Quercus pollen in Malaga (Spainsh Official Time).

Aunque el periodo de polinación del polen de Quercus en Málaga es relativamente estable, no sucede lo mismo con el momento en que se producen los picos máximos ni tampoco con los valores que alcanzan estos picos o las concentraciones anuales detectadas. Las mayores diferencias a este respecto se detectaron en los años 1992 y 1997, siendo en 1992 cuando el pico máximo presentó mayor retraso (Mayo), se alcanzó el máximo diario más bajo y se recogieron totales anuales más bajos, mientras que en 1997 el pico se adelantó al mes de Marzo y se alcanzaron las concentraciones máximas diarias y totales anuales más altos. Como indicaremos más adelante, en el apartado de correlación con parámetros meteorológicos, esos bajos valores y retraso para 1992 posiblemente se deban al efecto negativo de las precipitaciones primaverales (tab. 4).

Creemos que los periodos prolongados de sequía producen un debilitamiento del árbol traducido en pérdida de hojas, disminución del crecimiento vegetativo y, por tanto, de la posibilidad de formación de yemas florales y vegetativas (Cabezudo et al., 1993; Pérez Latorre et al., 1996; Navarro \& Cabezudo, 1998). Después de un periodo de sequía la presencia de un otoño lluvioso (1995) (tab. 4) no se traduce en un aumento en producción de yemas florales (1996) ni, por tanto, de polen (tab. 1) sino en una recuperación de la biomasa perdida, con predominio de la formación de yemas vegetativas y crecimiento de ramas (dolicoblastos). Sólo las lluvias otoñales del siguiente año (1996) (tab. 4) repercuten directamente en una producción masiva de yemas florales (1997) y, por tanto, de polen (tab. 1) y un adelanto en la floración producidos por condiciones óptimas de temperatura. Según Maldonado (1998), los meses de Febrero y Marzo de 1997 fueron muy secos y cálidos en comparación con otros años en casi todas las capitales de la península. En otras estaciones de muestreo españolas también se ha encontrado que, para Quercus, 1997 fue el año de polinación máxima y más adelantada (Cabezudo, ed., 1998a, 1998b). La disminución 
detectada en 1998 puede deberse a un periodo normal de descanso después de un agotamiento de los árboles por intensa producción de frutos.

Los diversos picos detectados a lo largo del PPP probablemente de debe a la floración escalonada de las distintas especies y poblaciones de Quercus presentes en el área de influencia del captador. La antesis se produce, de manera muy general, de Marzo a Junio fundamentamente, con variaciones fenológicas interanuales en función de variaciones climáticas, e intraanuales motivadas por las variaciones altitudinales en los hábitats de las especies. Las encinas presentan un intervalo de floración entre Febrero (litoral) y Junio (sierras); el alcornoque entre Abril y Julio, con un pico en Abril-Mayo; la coscoja tiene un intervalo fenológico entre Marzo y Julio, con pico en Abril-Mayo, y los quejigos presentan el intervalo entre Abril y Agosto, con pico de floración en Mayo-Junio (Cabezudo et al., 1993; Hidalgo \& Cabezudo, 1994; Pérez Latorre et al., 1996; Navarro \& Cabezudo, 1998).

En relación con el resto de los tipos polínicos registrados en Málaga, el de Quercus casi siempre ocupa el tercer lugar en orden de abundancia anual, después del de olivo y cupresáceas (Cabezudo et al., 1994; Toro et al., 1995; Recio et al., 1995, 1998; Trigo et al., 1998). Se trata, por tanto, de un taxon que presenta elevada incidencia en la atmósfera de Málaga. Tan sólo en 1996 pasó al octavo puesto, al tener especial relevancia los pólenes de gramíneas, plantagos y casuarinas (Trigo et al., 1998).

\section{Variación intradiaria}

Son muy escasos los trabajos que muestran el comportamiento intradiario del polen de Quercus. Tan sólo hemos encontrado los de Galán et al. (1991) para Córdoba, las recientes tesis doctorales de Alba (1997) y Munuera (1999) para Granada y Murcia respectivamente, además de los realizados para Málaga (Recio, 1995; Trigo et al., 1997) y Estepona (Toro, 1997). Cada uno de ellos han mostrado comportamientos diferentes. En todas estas ciudades las curvas tuvieron máximos poco acusados, inferiores al $15 \%$ del total, que se produjeron en diferentes momentos del día: generalmente por la tarde en Córdoba y Estepona, por la mañana en Murcia, y por la noche-madrugada en Málaga y Granada. También hemos encontrado, fuera de España, un trabajo (Käpylä, 1984) con resultados de variación intradiaria para el polen de roble (Quercus robur) comúnmente plantado en los parques de Turku (Finlandia), mostrando dos picos inferiores al $10 \%$, uno a mediodía y otro por la tarde, durante finales de mayo-principios de junio en los años 1977-79.

El modelo de distribución intradiaria obtenido para el polen de este taxon en Málaga presenta un comportamiento similar al obtenido para el polen de olivo (Recio et al., 1996; Trigo et al., 1997), con fluctuaciones que se suceden a lo largo del día, sin que las concentraciones máximas puedan asociarse a unas horas determinadas, y con una tendencia a incrementarse estas concentraciones polínicas durante la noche y la madrugada. Esta tendencia posiblemente sea debida a que, al igual que en el caso del olivo, la frecuencia de vientos del noroeste es mayor durante la noche (véase fig. 5 en Recio et al., 1996), y arrastran polen desde las zonas interiores hacia la costa, donde se sitúa la ciudad y el captador. Esta hipótesis se ve apoyada por los resultados obtenidos en las correlaciones, significativamente positivas con la velocidad del viento y la frecuencia de vientos del noroeste (tab. 3).

Por todo ello, aunque cabría pensar que el polen de Quercus debería concentrarse en el aire fundamentalmente durante las horas de mayor insolación y temperatura (mediodíatarde), pues es cuando se produciría la apertura de las anteras y la consiguiente liberación de sus pólenes al aire, dependiendo de la mayor o 


\begin{tabular}{lccccccc}
\hline & 1992 & 1993 & 1994 & 1995 & 1996 & 1997 & 1998 \\
\hline Horas de sol & 0,216 & 0,175 & 0,183 & $0,321^{* *}$ & $0,416^{* * *}$ & $0,373 * *$ & 0,012 \\
Precipitaciones & $0,228^{*}$ & $-0,006$ & 0,071 & $0,390^{* * *}$ & 0,116 & 0,206 & 0,132 \\
Temperatura media & 0,166 & $0,239^{*}$ & $0,235^{*}$ & 0,170 & $0,621^{* * *}$ & 0,193 & 0,009 \\
Temperatura máxima & $0,253^{*}$ & $0,276^{* *}$ & $0,233^{*}$ & $0,289^{* *}$ & $0,661 * * *$ & $0,311^{* *}$ & 0,099 \\
Temeperatura mínima & 0,037 & 0,166 & 0,135 & $-0,005$ & $0,442 * * *$ & $-0,014$ & $-0,098$ \\
Velocidad media viento & 0,072 & $0,501 * * *$ & $0,544 * * *$ & $0,382^{* * *}$ & 0,132 & $0,294^{*}$ & $0,387 * * *$ \\
\% viento $1^{\circ}$ cuadrante (NE) & 0,105 & 0,085 & $-0,042$ & 0,096 & 0,125 & 0,118 & $-0,244 *$ \\
\% viento $2^{\circ}$ cuadrante (SE) & $-0,031$ & $-0,237 *$ & $-0,390^{* * *}$ & $-0,439 * * *$ & $-0,117$ & $-0,244 *$ & $-0,396^{* * *}$ \\
\% viento $3^{\circ}$ cuadrante (SO) & $-0,033$ & $-0,200^{*}$ & $-0,366^{* *}$ & $-0,123$ & 0,066 & 0,021 & 0,175 \\
\% viento $4^{\circ}$ cuadrante (NO) & 0,075 & $0,310^{* *}$ & $0,397 * * *$ & $0,509 * * *$ & 0,097 & $0,319 * *$ & $0,314 * *$ \\
\hline
\end{tabular}

Tabla 3. Coeficientes de correlación de Spearman obtenidos entre los valores diarios de concentración de polen de Quercus, expresados en $\mathrm{N}^{\circ}$ granos de polen $/ \mathrm{m}^{3}$, y algunos parámetros meteorológicos durante el período de polinación principal. Spearman's correlation coefficients obtained between daily values of Quercus airborne pollen, expressed in No. pollen grains/ $\mathrm{m}^{3}$, and the principalmeteorological parameters during the main pollen season (PPP).

\begin{tabular}{lccccccc}
\hline & $1991-92$ & $1992-93$ & $1993-94$ & $1994-95$ & $1995-96$ & $1996-97$ & $1997-98$ \\
\hline Otoño & 243,3 & 159,4 & 200,6 & 81,4 & 253,2 & 428,7 & 425,7 \\
Invierno & 62,8 & 152,6 & 164,5 & 51,5 & 522,6 & 406,8 & 285,8 \\
Primavera & 104,8 & 76,4 & 45,3 & 3,2 & 153,7 & 93,5 & 82,7 \\
Verano & 1,8 & 1,2 & 3,3 & 12,0 & 46,5 & 6,1 & 3,7 \\
\hline Total & 412,7 & 389,6 & 413,7 & 148,1 & 976,0 & 935,1 & 797,9 \\
\hline
\end{tabular}

Tabla 4. Precipitaciones registradas en Málaga durante las cuatro estaciones en los años de estudio. Los datos corresponden a años hidrológicos. Accumulated rainfall recorded in Malaga during the different seasons (autumn, winter, spring and summer) during1991-1998.

menor cercanía al captador de las especies de Quercus, su comportamiento a va ser diferente, desplazándose el pico hacia las horas en que las corrientes de aire transportan las nubes de polen hacia el captador, en este caso situado en la ciudad.

Si se comparan los IDI obtenidos por el polen de Quercus con el de otros tipos polínices observaremos que se trata de un taxon que, al menos en Málaga, presenta siempre valores muy bajos. Este tipo de comportamiento intradiario, con valores bajos del IDI, ya había sido interpretado como un indicio de transporte a cierta distancia (Trigo et al., 1997).

\section{Correlación con parámetros meteorológicos}

La polinación de este taxon presenta un comportamiento bastante estable en relación con determinados factores meteorológicos, pues se ha observado que los incrementos de concentraciones polínicas siempre estuvieron asociados a incrementos de calor (temperatura e insolación) y de velocidad de viento, y que la dirección del viento predominante era la del cuarto cuadrante (noroeste). Käpylä (1984) también encontró para Turku (Finlandia) correlaciones positivas entre las concentraciones polínicas de este taxon y la 
temperatura y velocidad del viento.

Nuestras correlaciones obtenidas con los vientos parecen indicar que la mayoría de los pólenes de Quercus registrados en la ciudad de Málaga proceden de las sierras del interior, donde se encuentran las grandes masas de encinares y alcornocales, y donde es posible que alcancen niveles superiores a los detectados en Málaga.

En los años 1992 y 1996 no se obtuvieron correlaciónes significativas con ningún parámetro relacionado con viento. Fueron años de mínimos registros anuales de polen de este género (tab. 1) y de primaveras más lluviosas (tab. 4), lo que nos hace pensar que el que no se detectaran altas concentraciones de este tipo polínico posiblemente sea debido a esas lluvias primaverales que, por efecto de lavado atmosférico, hicieron descender las concentraciones, a pesar de ser durante esta estación cuando las inflorescencias masculinas se encuentran repletas de granos de polen a punto de ser dispersados por el viento. En estos dos años, el efecto de las precipitaciones hicieron que, por mucho viento favorable o desfavorable que tuvieramos, no se haya obtenido correlación significativa alguna. Por otra parte, creemos que la precipitación influye de manera positiva fundamentalmente en la formación de las yemas florales y no en la apertura y maduración de las flores.

\section{CONCLUSIONES}

El polen de Quercus se detecta en la atmósfera de Málaga fundamentalmente durante los meses de Marzo, Abril y Mayo, registrándose generalmente las mayores concentraciones durante el mes de Abril. Otros picos de menor intensidad pueden producirse en Mayo, debido a la polinación de especies con distinta fenología que habitan en zonas más elevadas o interiores de la provincia.

Las cantidades anuales y el valor de los picos máximos son variables, siendo 1992 y 1997 los años en los que se registraron los valores más bajos y más altos, respectivamente, de polen de Quercus.

En Málaga, el polen de Quercus representa generalmente el tercer tipo polínico en orden de importancia anual, después del de olivo y del de cupresáceas.

Al igual que en otras estaciones de la Red Española de Aerobiología, 1997 fue el año en que se obtuvieron los máximos registros y polinación más adelantada. A ello parece ser que contribuyeron las intensas lluvias otoñales, previas al período de polinación, acaecidas después de un fuerte periodo de sequía, lo que se tradujo en la formación de un mayor número de yemas florales; así como las altas temperaturas que se alcanzaron durante el invierno (Febrero-Marzo), que contribuyeron a una pronta maduración de las yemas ya formadas.

La distribución intradiaria de este tipo polínico es muy variable, aunque se observa para Málaga una ligera tendencia a incrementarse las concentraciones durante la noche y la madrugada. Este comportamiento, indicativo de transporte a larga distancia, está posiblemente relacionado con la fuerte dependencia de determinados parámetros meteorológicos, que se indican a continuación.

Las variables meteorológicas que significativamente se asocian con concentración atmosférica de polen de Quercus en Málaga son, de manera positiva, los parámetros indicadores de calor (temperatura e insolación) así como la velocidad y frecuencia noroeste del viento, mientras que las calmas y las restantes direcciones de viento se asocian negativamente. Estas correlaciones obtenidas con viento parecen indicar que la mayoría de los pólenes de Quercus que se registran en Málaga proceden de las sierras del interior, donde se encuentran las mejores poblaciones de encinas, alcornoques y coscojas, y que son arrastrados por los vientos del noroeste hacia la capital costera. 
AGRADECIMIENTOS. Los autores desean agradecer a las Consejerías de Salud y Educación de la Junta de Andalucía la colaboración prestada a la Red Andaluza de Aerobiología (RAA) para el desarrollo de este trabajo.

\section{BIBLIOGRAFÍA}

ALBA, F. -1997- Caracterización polínica de la atmósfera de Granada: relación con las variables meteorológicas y modelos predictivos de los taxones más alergógenos. Tesis Doctoral. Universidad de Granada.

BERMEJO, D., A. M. GARCÍA, P. SANCHO y C. VALERO -1998a- Aerobiología en Aragón: estación de Zaragoza (1995-1996). Rea 3: 45-48.

BERMEJO, D., A. M. GARCÍA, P. SANCHO y C. VALERO -1998b- Aerobiología en Aragón: estación de Zaragoza (1997). Rea 4: 53-56.

CABEZudo, B. (ed.) -1998a- Boletín de la Red Española de Aerobiología. Años 1995-1996. Rea 3: 1-100.

CABEZUDO, B. (ed.) -1998b- Boletín de la Red Española de Aerobiología. Año 1997. Rea 4: 1132.

CABEZUdo, B., A. V. PÉREZ latorRe, T. NAVARRO y J. M. NIETO CALDERA - 1993Estudios fenomorfológicos en la vegetación del Sur de España. II. Alcornocales mesomediterráneos. (Montes de Málaga, Málaga). Acta Bot. Malacitana 18: 179-188.

CABEZUDO, B., M. M. TRIGO, M. RECIO y F. J. TORO -1994- Contenido polínico de la atmósfera de Málaga: Años 1992 y 1993. Acta Bot. Malacitana 19: 137-144

DÍEZ, M. J. y R. SUÁREZ - 1987- Fagaceae. En: B. VALDES, M. J. DÍEZ \& Y. FERNÁNDEZ (eds.) Atlas Polínico de Andalucía Occidental. Instituto de Desarrollo Regional de la Universidad de Sevilla. Utrera, Sevilla.

DOMÍNGUEZ VILCHES, E. -1995- La Red Española de Aerobiología (REA). Rea 1: 3-7.

DOMÍNGUEZ VILCHES, E., J. L. UBERA y C. GALÁN -1984-Polen alergógeno de Córdoba. Publicaciones del Monte Piedad y Caja de Ahorros de Ronda. Córdoba.

DOMÍNGUEZ VILCHES, E., C. GALÁN, F. VILLAMANDOS y F. INFANTE - 1991Handling and evaluation of the data from the aerobiological sampling. Monografía REA/ EAN 1: 1-18. Universidad de Córdoba.

ERIKSSON, N. E. -1978- Allergy to pollen from different decidous trees in Sweden. Allergy 33: 299-309.

FERNÁNDEZ CALDAS, E., M. C. SWANSON, J. PRAVDA, P. WELSH, J. W. YUNGINGER \& C. E. REED -1989-Immunochemical demonstration of red oak pollen aeroallergens outside the oak pollination season. Grana 28: 205-209.

GALÁN, C. R. TORMO, J. CUEVAS, F. INFANTE \& E. DOMÍNGUEZ -1991- Theoretical daily variation patterns of airborne pollen in the South-West of Spain. Grana 30: 201-209.

HALSE, R. R. - 1984- Nomenclature of Allergenic Plants. Ann. Allergy 53: 291-307.

HIDALGO, M. I. y B. CABEZUDO -1994Fenología y volumen de floración del matorral de la Sierra de Mijas (Málaga, S. España). Acta Bot. Malacitana 19: 123-136.

HIDALGO, M. I., B. CABEZUDO y M. RECIO 1996a- Producción floral en un matorral del Sur de España. Anales Jard. Bot. Madrid 54(1): 547-553.

HIDALGO, M. I., M. RECIO y B. CABEZUDO 1996b- Producción de polen en un matorral del Sur de España. Acta Bot. Malacitana 21: 49-55. HIRST, J. M. -1952- An automatic volumetric spore trap. Ann. Appl. Biol. 39(2): 257-265.

ICKOVIC, M. R. \& M. THIBAUDON -1991Allergenic significance of Fagaceae pollen. In:G. D'AMATO, T. TH. M. SPIEKSMA \& S. BONINI (eds.) Allergenic Pollen and Pollinosis in Europe, pp. 98-108. Blackwell Scientific Publications. Oxford.

KÄPYLÄ, M. - 1984- Diurnal variation of tree pollen in the air in Finland. Grana 23: 167-176.

KORMUTÁK, A. \& M. G. OSTROLUCKÁ - 1992 Serological properties of the pollen proteins in some Quercus species. Biologia (Bratislava) 47(4): 281-285.

LEWIS, W. H., P. VINAY \& V. E. ZENGER -1983Airborne and allergenic pollen of North America. Hopkins, London.

MALDONADO, J. A. -1998- Resumen climático del año 1997. Rea 4: 7-9.

MUNUERA, M. -1999- Patrones de variación polinica en la atmósfera de Murcia. Implicaciones alergológicas, prevención y 
diagnóstico. Tesis Doctoral. Universidad de Murcia.

NAVARRO, T. y B. CABEZUDO - 1998-Estrategias fenomorfológicas de especies de un matorral mediterráneo (Andalucía, España). Acta. Bot. Malacitana 23: 133-148.

NILSSON, S. \& F. TH. M. SPIEKSMA (eds.) 1992- Traveller's Allergy Service Guide. Swedish Museum of Natural History and Fisons Sweden AB, Sweden.

PATHIRANE, L. -1975- Graphical determination of the main pollen season. Pollen et Spores 17(4): 609-610.

PÉREZ LA TORRE, A. V., B. CABEZUDO, J. M. NIETO CALDERA y T. NAVARRO -1996Caracterización fenológica y ecomorfológica de alcornocales andaluces (Málaga, España). Anales Jard. Bot. Madrid 54(1): 554-560.

RECIO, M. -1995- Calendario polínico de la atmósfera de Málaga (1991-1994). Relación con los parámetros meteorológicos. Tesis Doctoral. Universidad de Málaga.

RECIO, M., M. M. TRIGO, F. J. TORO y B. CABEZUDO -1995- Contenido polínico de la atmósfera de Málaga: Año 1994. Acta Bot. Malacitana 20: 83-90.

RECIO, M., B. CABEZUDO, M. M. TRIGO \& F. J. TORO -1996- Olea europaea pollen in the atmosphere of Málaga (S. Spain) and its relationship with meteorological parameters. Grana 35: 308-313.

RECIO, M. M. M. TRIGO, F. J. TORO y B. CABEZUDO -1997- Incidencia del polen de Plantago en la atmósfera de Málaga y su relación con los parámetros meteorológicos. Acta Bot. Malacitana 22: 103-113.

RECIO, M., B. CABEZUDO, M. M. TRIGO \& F. J. TORO - 1998a- Pollen calendar of Malaga (Southern Spain), 1991-1995. Aerobiologia 14: 101-107.

RECIO, M., M. M. TRIGO, F. J. TORO y B. CABEZUDO - 1998b- Aerobiología en Andalucía: Estación de Málaga (1997). Rea 4: 41-44.

ROSS, A. M., J. M. CORDEN \& D. M. FLEMING -1996- The role of oak pollen in hay fever consultations in general practice and the factors influencing patient's decisions to consults. Brit. J. Gen. Pract. 46: 451-455.

SÁENZ, C. -1978- Polen y Esporas (Introducción a la Palinología y vocabulario palinológico).
Blume. Madrid.

SPIEKSMA, F. TH. M. -1991-Regional European Pollen Calendars In: G. D’AMATO, F. TH. M. SPIEKSMA \& S. BONINI (eds.) Allergenic Pollen and Pollinosis in Europe, pp. 49-65. Blackwell Sientific Publications, Oxford.

Sutra, J. P., G. PElTRE, A. LAPAlus, P. JULIEN, P. RUFFALDI, G. SULMONT \& J. LAFOND -1990- Aeropalynological and immunochemical data on Fagales pollen grains. Rev. Paleobot. Palynol. 64: 53-59.

TORO, F. J. -1997-Estudio aerobiológico de la Costa del Sol Occidental: Málaga y Estepona (1995-1997). Análisis comparativo y modelos de pronóstico. Teisis Doctoral. Universidad de Málaga.

TORO, F. J., M. RECIO, M. M. TRIGO y B. CABEZUDO -1996- Contenido polínico de la atmósfera de Málaga: Año 1995. Acta Bot. Malacitana 21: 57-63.

TORO, F. J., M. RECIO, M. M. TRIGO \& B. CABEZUDO -1998a- Predictive models in aerobiology: data transformation. Aerobiologia 14: 179-184.

TRIGO, M. M., M. RECIO, F. J. TORO \& B. CABEZUDO - 1997- Intradiurnal fluctuations in airborne pollen in Málaga (S. Spain): a quantitative method. Grana 36: 39-43.

TRIGO, M. M., M. RECIO, F. J. TORO y B. CABEZUDO - 1998- Aerobiología en Andalucía: estación de Málaga (1995-1996). Rea 3: 33-36.

Aceptado para su publicación en Septiembre de 1999

Dirección de los autores. Dpto. Biología Vegetal. Fac. Ciencias. Campus de Teatinos. Universidad de Málaga. e-mail: aerox@uma.es 